\title{
ANALYTICAL DESIGN OF A WAVEGUIDE IRIS/STUB TUNING COUPLER TO AN OVERCOUPLED SUPERCONDUCTING CAVITY
}

\author{
Haipeng Wang* \\ TJNAF, Newport News, VA 23606, USA
}

\begin{abstract}
The high RF power test of a superconducting cavity without beam loading requires a variable coupling scheme. For a fixed power coupler and a finite source power, one needs to increase the external Q. A 3-stub tuner placed between the doorknob transition and an iris plate inside of WR975 waveguide has been developed for the SNS cryomodule test facility (CMTF). This paper describes the theoretical analysis, calculation by equivalent circuits and the coupling scale related to the stub tuning and the iris size. A copper model bench measurement and a $3 \mathrm{D}$ computer simulation proved the principle and some of the parameters. A factor of 100 over $\mathrm{Q}_{\mathrm{ext}}=7 \times 10^{5}$ has been achieved when implemented in the cryomodule test.
\end{abstract}

\section{INTRODUCTION}

The external quality factor $\mathrm{Q}_{\mathrm{s}, \mathrm{ext}}$ of the fundamental power coupler (FPC) to a superconducting cavity is normally designed much lower than the cavity's intrinsic quality factor $\mathrm{Q}_{\mathrm{s}}$ in order to match the input power to the beam load. For example, $\mathrm{Q}_{\mathrm{s}, \mathrm{ext}}=10^{5} \sim 10^{7}, \mathrm{Q}_{\mathrm{s}}=10^{9} \sim 10^{10}$. That means the coupling co-efficient $\beta_{\mathrm{s}}=\mathrm{Q}_{\mathrm{s}} / \mathrm{Q}_{\mathrm{s}, \mathrm{ext}}$ from FPC to cavity is always heavily over-coupled $\beta_{\mathrm{s}}>>1$. A common high power type FPC was also designed in a fixed coupling in order to handle a high power and save cost. When the cavity cryomodule is under the final high power test without a beam loading, most of forward power is going to reflect back and to dump into the klystron's circulator. Using a finite klystron power to achieve a high gradient field in the cavity, one needs to increase the equivalent external $\mathrm{Q}\left(\mathrm{Q}_{\mathrm{eq}, \mathrm{ext}}\right)$ by $1 \sim 3$ order of magnitude, up to the maximum level of microphonics. A simple and common solution is to use a 3-stub tuner on the input power waveguide. Normally this tuning mechanism can only change $Q_{\text {eq,ext }}$ about 1 order. By adding an iris plate upstream to klystron, like in the Figures $1 \& 2$, from the following analysis, one can get 2 orders higher. Combining the 3 -stub tuning capability, the coupling of $\beta_{\mathrm{eq}}=\mathrm{Q}_{s} / \mathrm{Q}_{\mathrm{eq}, \mathrm{ext}}$ can be variable. Here we define loaded $\mathrm{Q}_{\mathrm{sl}}=\mathrm{Q}_{\mathrm{s}} /\left(1+\beta_{\mathrm{eq}}\right)$. This mechanism is very attractive for an energy recovery recirculating linac (ERL). When the beam energy is returned to the cavity, the beam loading is gone. A higher external Q will save more klystron power from its circulator. Using a variable coupling, a same cavity can be used for both beam acceleration and storage. It can also help in helium

* email: haipeng@jlab.org. processing to bring more power to the cavity for destroying the field emitters. In the CMTF, using a 3-stub tuner only was the usual case for small Q external changes.

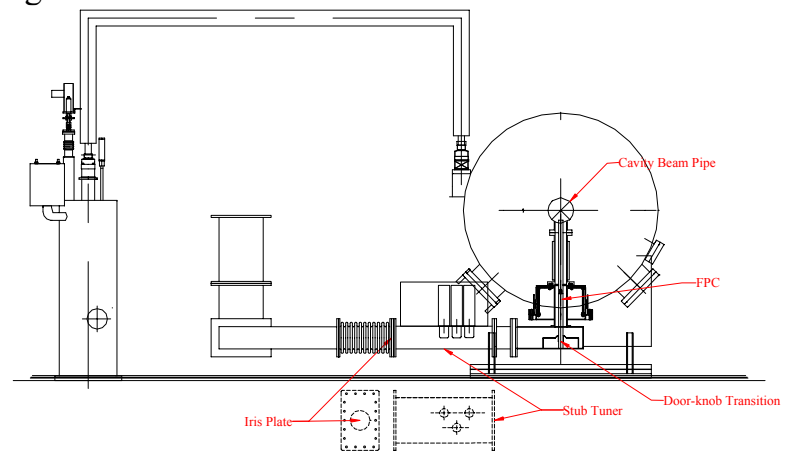

Figure 1: Iris/stub tuner design layout for SNS cryomodule tests.

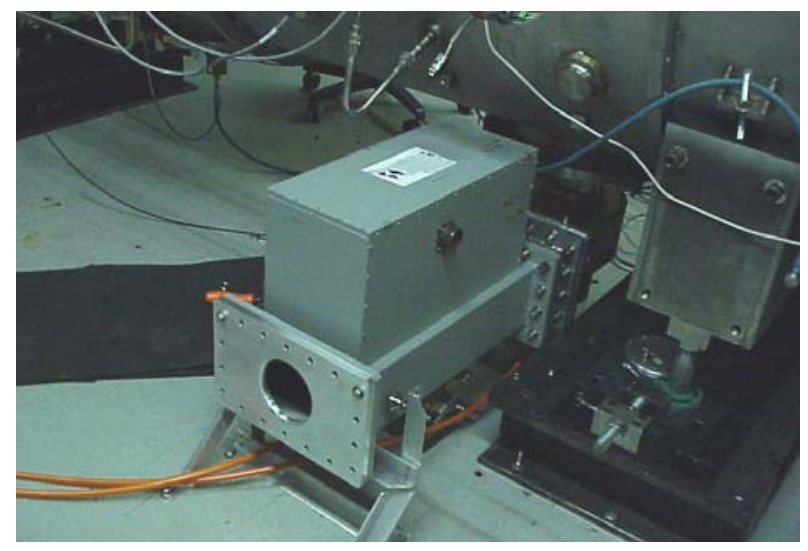

Figure 2: An iris plate installed next to the stub tuner for a $15 \mathrm{~kW}$ CW klystron system at TJNAF.

\section{EQUIVALENT CIRCUIT ANALYSIS}

The waveguide section between the iris plate and the superconducting (sc) cavity detuning short can be treated as a low-Q coupling resonator. The power coupling from klystron to the sc cavity will be two-stage impedance transforming (see Figure 3). The sc cavity loaded Q can be deduced as [1]:

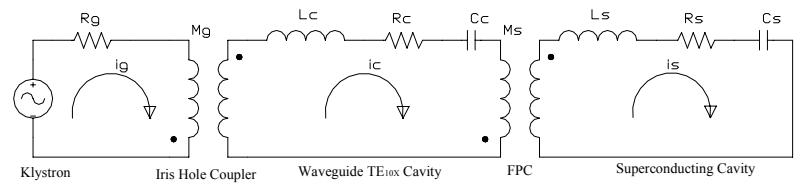

Figure 3: Resonator coupling equivalent circuit. 


$$
Q_{S L}=\frac{Q_{s}\left(1+\beta_{g}\right)-Q_{s} \delta_{s}+Q_{c}-Q_{c} \delta_{c}}{1+\beta_{g}+\beta_{s}-Q_{s} \delta_{s} Q_{c} \delta_{c}}
$$

Here

$$
\begin{aligned}
& \omega_{c}^{2}=\frac{1}{L_{c} C_{c}} \quad \omega_{s}^{2}=\frac{1}{L_{s} C_{s}} \quad Q_{c}=\frac{\omega L_{c}}{R_{c}} \quad Q_{s}=\frac{\omega L_{s}}{R_{s}} \\
& \beta_{g}=\frac{\omega^{2} M_{g}^{2}}{R_{c} R_{g}} \quad \beta_{s}=\frac{\omega^{2} M_{s}^{2}}{R_{c} R_{s}} \quad \delta_{c}=1-\frac{\omega_{c}^{2}}{\omega^{2}} \quad \delta_{s}=1-\frac{\omega_{s}^{2}}{\omega^{2}}
\end{aligned}
$$

Using the fact that mostly $\mathrm{Q}_{\mathrm{s}}>>\mathrm{Q}_{\mathrm{c}}>>\mathrm{Q}_{\mathrm{s}} \delta_{\mathrm{s}}>>\mathrm{Q}_{c} \delta_{\mathrm{c}}$. (1) can be simplified as:

$$
\frac{Q_{e q, e x t}}{Q_{s, e x t}}=\frac{\beta_{s}}{\beta_{e q}}=\frac{1+\beta_{g}}{1-\frac{Q_{s} \delta_{s} Q_{c} \delta_{c}}{\beta_{s}}}=\frac{1+Q_{c} / Q_{c, e x t}}{1-Q_{s, e x t} \delta_{s} Q_{c} \delta_{c}}
$$

We define the $\mathrm{Q}_{\mathrm{c}}$ is the coupling cavity intrinsic $\mathrm{Q} . \mathrm{Q}_{\mathrm{c}, \mathrm{ext}}$ is the iris coupling external $\mathrm{Q}$ to the coupling cavity. The relative frequency detuning for sc cavity $\delta_{\mathrm{s}}$ is normally limited by the static tuner and microphonics' level. However, stub tuning can change the relative frequency of coupling cavity $\delta_{\mathrm{c}}$, so as to change the $\mathrm{Q}_{\text {eq,ext }} / \mathrm{Q}_{\mathrm{s} \text {,ext }}$. Note that this detuning requires $\mathrm{Q}_{\mathrm{s}, \mathrm{ext}} \delta_{\mathrm{s}} \mathrm{Q}_{\mathrm{c}} \delta_{\mathrm{c}}<1$. That means stub frequency detuning for the resonance coupling condition has to be:

$$
\Delta f_{c}<\frac{f^{2}}{4 Q_{s, e x t} Q_{c} \Delta f_{s}}
$$

Here $f$ is the klystron operating frequency. For example, for a SNS medium beta cavity, $\mathrm{Q}_{\mathrm{s}, \mathrm{ext}}=7.3 \times 10^{5}, \mathrm{f}=805 \mathrm{MHz}$, assumed $\Delta \mathrm{f}_{\mathrm{s}}=10 \mathrm{~Hz}, \mathrm{Q}_{\mathrm{c}}=1 \times 10^{3} . \Delta \mathrm{f}_{\mathrm{c}}$ must be less than $22 \mathrm{MHz}$. The $\mathrm{Q}_{\mathrm{c}}$ determines the sensitivity of stub tuning. If the $Q_{c}$ can be made high enough, a fast tuner can be used to compensate the Lorenz force detuning and microphonics noise. From Equation (4), we can see that this iris/stub tuning coupler can only increase equivalent external $\mathrm{Q}$ or $\mathrm{Q}_{\mathrm{eq}, \mathrm{ex}}>\mathrm{Q}_{\mathrm{s}, \mathrm{ext}}$.

People worry about the power loss of coupling cavity $\mathrm{P}_{\mathrm{c}}$ relative to the power loss in the sc cavity $\mathrm{P}_{\mathrm{s}}$. It can be found out [1] that it mainly depends on the sc cavity's microphonics' level. Figure 3 shows this calculation.

$$
\frac{\boldsymbol{P}_{c}}{\boldsymbol{P}_{s}}=\frac{\boldsymbol{Q}_{s, \mathrm{ext}}}{\boldsymbol{Q}_{s}}\left(1+4 \boldsymbol{Q}_{s}{ }^{2} \frac{\Delta f_{s}^{2}}{f^{2}}\right)
$$

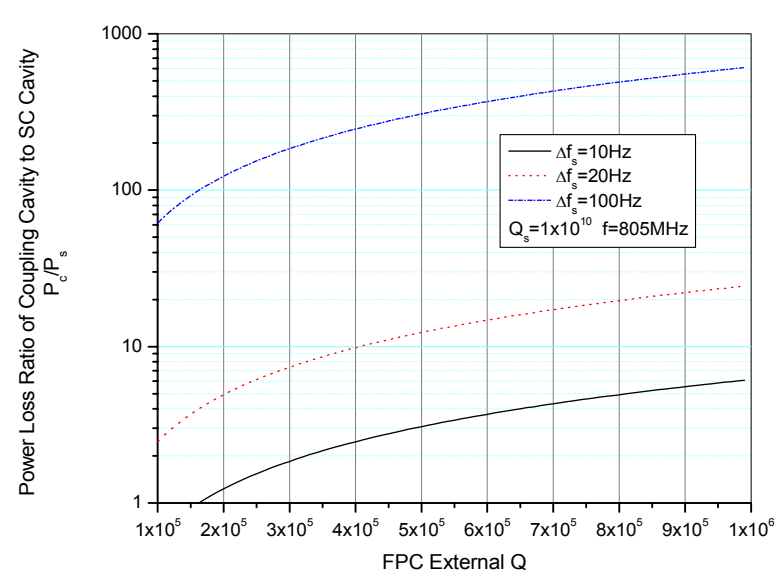

Figure 3. Power loss ratio in the coupling cavity.
The $\mathrm{Q}_{\mathrm{c}, \mathrm{ext}}$ in Equation (4) depends on the iris hole's size, position and thickness. From the analytical calculation of next section, the external Q enhancement factor can be plotted as the function of iris diameter (Figure 4).

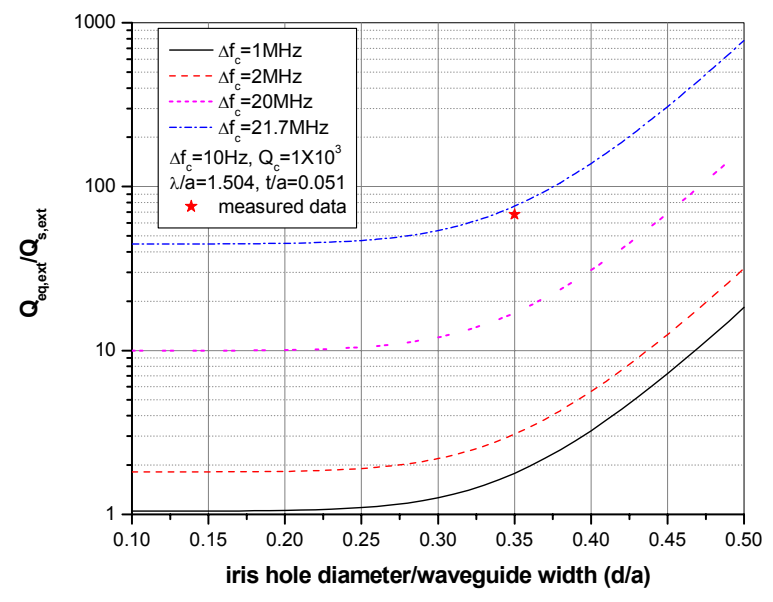

Figure 4: Analytical calculation verse one measured data.

\section{IRIS COUPLING CALCULATION}

The iris hole coupling to a rectangular waveguide $\mathrm{Q}_{\mathrm{c}, \mathrm{ext}}$ can be done by a 3D computer simulation like MAFIA or Analyst (Omega3). The first approach I took is the analytical solution. The susceptance of an iris inside of waveguide is [2]:

$$
\frac{B}{Y_{0}}=\frac{\lambda_{g}}{a}\left[\frac{\pi b}{24 d j_{1}{ }^{2}}-1+A_{1}-\left(\frac{a}{\lambda}\right)^{2} A_{2}\right]
$$

With an iris thickness (Figure 5) correction:

$$
\frac{B_{a}}{Y_{0}}=\frac{B}{2 Y_{0}}+\frac{\left|Y_{0}^{\prime}\right|}{Y_{0}} \tanh \frac{\pi t}{\left|\lambda_{g}^{\prime}\right|} \quad \frac{B_{b}}{Y_{0}}=\frac{\left|Y_{0}^{\prime}\right|}{Y_{0}} \operatorname{csch} \frac{2 \pi t}{\left|\lambda_{g}^{\prime}\right|}
$$

Here Y0 is the shunt conductance of waveguide. The $j_{1}$, $\mathrm{A}_{1}, \mathrm{~A}_{2}$, and $\mathrm{Y}_{0}{ }^{\prime}, \lambda_{\mathrm{g}}{ }^{\prime}$ are all the functions of $\mathrm{d} / \mathrm{a}$, see Ref. [2] for detail.

Q external of an iris coupling to a TE10x rectangular cavity can be deduced from Ref. [3] by the circuit transformation of Figure 6:

$$
Q_{e x t}=\left(\frac{B}{Y_{0}}\right)^{2}\left[\frac{\pi}{2} \frac{1}{1-(\lambda / 2 a)^{2}}+\frac{Y_{0}}{B}\right]
$$

With an iris thickness correction:

$$
Q_{c, e x t}=\left(\frac{B_{a}}{Y_{0}}\right)^{2} \frac{\pi}{2} \frac{1}{1-\left(\frac{\lambda}{2 a}\right)^{2}}\left(\frac{B_{a}+2 B_{b}}{B_{b}}\right)^{2}
$$

There is another formula from Collin [4] for the case of without iris thickness:

$$
Q_{c, e x t}=\frac{9}{4 \pi} \frac{b^{2} c\left(a^{2}+c^{2}\right) \lambda}{d^{6} \sqrt{1-(\lambda / 2 c)^{2}}}
$$

Here $\mathrm{a}, \mathrm{b}$ and $\mathrm{c}$ is waveguide width, height and length respectively. The $\mathrm{d}$ is the iris hole diameter.

In Figure 7, I compare two analytical methods, MIT's Equations (7) (10) and Collin's Equation (11), with a 
Analyst 3D simulation [5]. The simulation used Balleyguier's method [6]. The simulation has confirmed
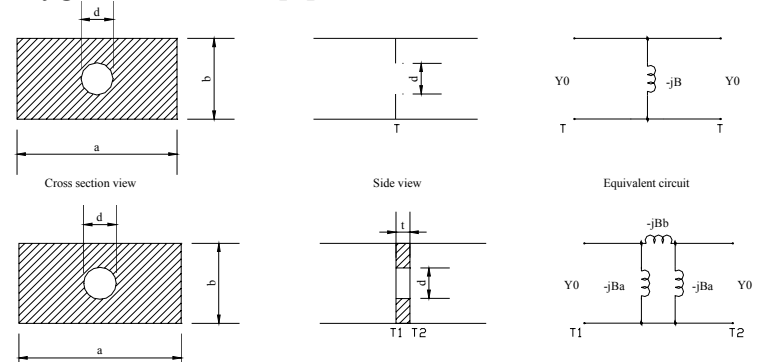

Figure 5: Iris without (top) and with (bottom) thickness simulation inside of a TE10 rectangular waveguide.

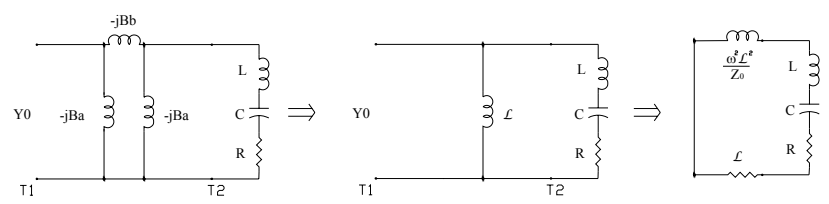

Figure 6: Equivalent circuit transformation from an iriscoupled to a short-circuit waveguide cavity.

that the detail of meshing around the iris hole is important. The thickness of the iris has also a significant susceptance contributed to the $\mathrm{Q}_{\mathrm{c}, \mathrm{ext}}$ 's calculation.

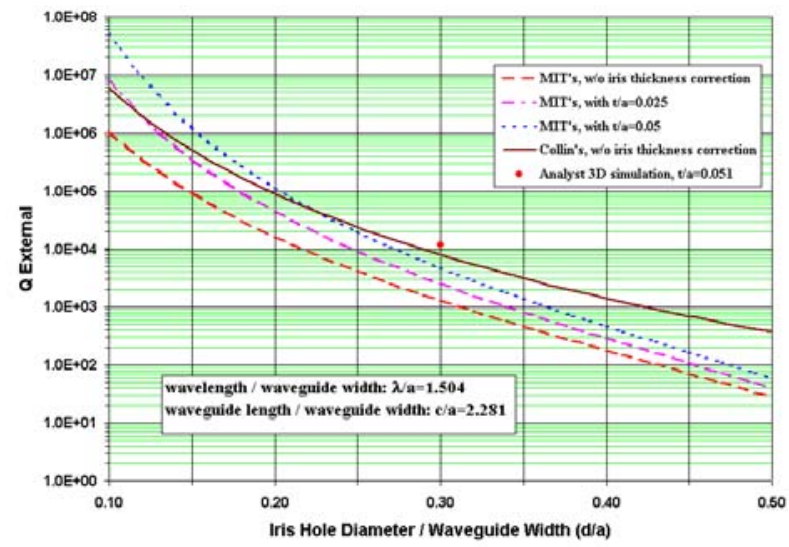

Figure 7: Q external calculation for an iris hole coupling to a TE10x rectangular waveguide resonator.

\section{IRIS POSITION DETERMINATION}

The position of the iris plate along the waveguide has to be at the voltage minima of standing wave (SW) when the cavity/coupling system is on resonance. So the iris inductance will be effectively at the maximum SW current position. Also the coupling waveguide will be in the TE01n mode resonant frequency when the waveguide length $\mathrm{c}=\mathrm{n} \lambda_{\mathrm{g}} / 2$. For a waveguide coupling to a normal conducting cavity, like a copper or a room temperature niobium cavity, the "detuned short" position is also the voltage minimum position in resonance. It can be explained by the voltage reflection coefficient, because of $\beta<<1$ :

$$
\Gamma(\omega)=\frac{\beta-1-i Q_{0} \delta}{\beta+1+i Q_{0} \delta} \quad \delta=\frac{\omega}{\omega_{0}}-\frac{\omega_{0}}{\omega}
$$

When the cavity changes from normal conducting state to superconducting state, the coupling coefficient changes from $\beta<<1$ to $\beta>>1$. The voltage minimum position at resonance will be changed by $\lambda_{\mathrm{g}} / 4\left(90^{\circ}\right)$. It is important to incorporate this change in the design. For the SNS medium beta cavity coupling, the iris position was determined by a computer 3D simulation, then by a bench measurement on a model. Using a slot-line waveguide (or a hole array on the centerline) coupled to a copper cavity, I measured the SW profile. From the plot in Figure 8, I got that the distance from the door-knob center to the first $\mathrm{V}_{\min }$ is $52.6 \mathrm{~cm}$. The HFSS simulation from Y. Kang is $52.7 \mathrm{~cm}$ [7]. Both of them are for the copper cavity. The iris position in the actual design was pulled back by $3 / 4 \lambda_{\mathrm{g}}$.

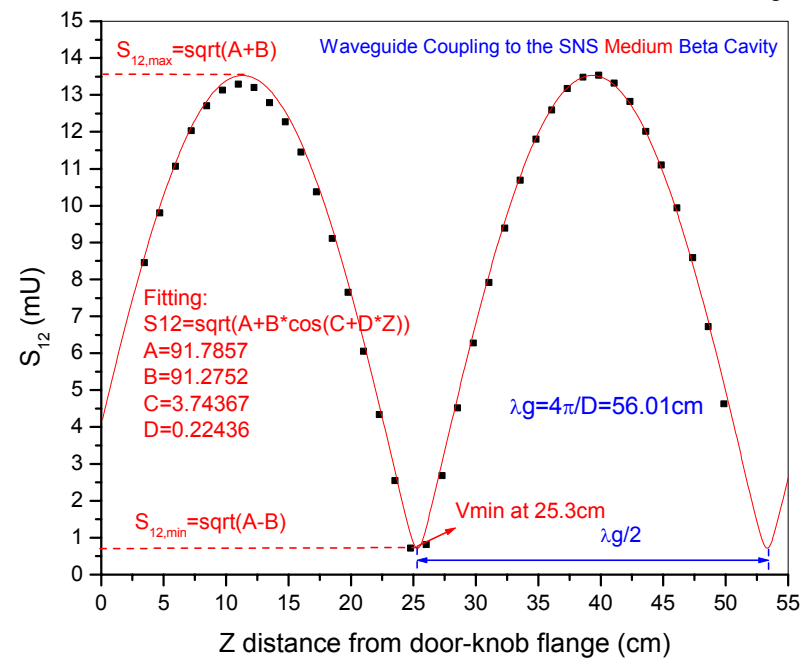

Figure 8: SW profile measured on WR975 waveguide.

\section{SUMMARY}

The combination of iris plate and 3-stub tuner can be used as a variable coupler. It increases the equivalent external Q of an over-coupled superconducting cavity by two orders of higher. More measurement needs to be done to verify this analysis when the CMTF is available for this study.

I would like to thank R. Sundelin, L. Phillips, C. Reece, and J, Delayen, I. Campisi and Y. Kang of ORNL for the fruitful discussions during the course of this work.

\section{REFERENCE}

[1] I. Ben-Zvi, SUNYLAC Internal Report, April 1980.

[2] Waveguide Handbook, edited by N. Marcuvitz, MIT Radiation Lab, 1986 edition, p238 240, p327, p408 409.

[3] Principle of Microwave Circuits, edited by C. Montgomery, R. Dicke, E. Purcell, MIT Radiation Lab, series 1948, p231 234.

[4] Field Theory of Guided Waves, second edition, R. Collin, p523 531.

[5] Private communication with J. DeFords of STAR, Inc.

[6] P. Balleyguier, LINAC98 proceedings, MO4037.

[7] Private communication with Y. Kang of ORNL. 